\title{
Do ventricular repolarization interval ratios depend on heart rate and should they be rate-corrected?
}

\author{
Carlos Alvarado-Serrano ${ }^{1,2}$, Juan Ramos-Castro ${ }^{2}$, and Ramon Pallàs-Areny ${ }^{2}$ \\ ${ }^{1}$ Department of Electrical Engineering, CINVESTAV-IPN, México D.F., México \\ ${ }^{2}$ Department of Electronic Engineering, Technical University of Catalonia (UPC), Barcelona, Spain
}

\begin{abstract}
QT interval decreases with increasing heart rate (HR), hence to define normal values of QT interval for different heart rates requires the application of a $Q T$ interval rate correction formula. However, it's unknown the influence of $H R$ on the ratios between several ventricular repolarization intervals, that can be useful as risk predictors of malignant ventricular arrhythmias. This study analyzes the influence of HR on QTp/QT, JTp/JT, Tpe/JTp, and Tpe/JT ratios on a healthy subject during exercise. The intervals were measured in V3 lead and the characteristic points of QRS complex and $T$ wave were detected by computer algorithms based on the wavelet transform (WT). Exercise stress was graded according to the four initial stages of the Bruce protocol. The heart rate was divided into two ranges: less than 100 beats/min and from 100 beats $/ \mathrm{min}$ to 185 beats/min. When HR exceeded 100 beats/min, QTp/QT and $\mathbf{J T p} / \mathrm{JT}$ ratios increased whereas $\mathrm{Tpe} / \mathrm{JTp}$ and $\mathrm{Tpe} / \mathrm{JT}$ ratios decreased for increasing HR. However, when HR was below 100 beats $/ \mathrm{min}$, those same four ratios were independent from HR. As opposed to the QT interval, there is no need to correct QTp/QT, JTp/JT, Tpe/JTp, and Tpe/JT ratios when $\mathrm{HR}$ is below 100 beats/min.
\end{abstract}

Key words-QT interval, exercise ECG, heart rate

\section{INTRODUCTION}

QT prolongation in the 12-lead ECG is associated with a high risk of ventricular arrhythmias and sudden cardiac death in various clinical conditions, for example in postmyocardial infarction (MI) patients, however, its predictive value is controversial [1,2]. The discrepancies about the prognostic value can be attributed to that the QT interval has many sources of variation [3]. One of them are the measurement methods that use different definitions for $\mathrm{T}$ wave end and different lead. Other sources of variation are due to physiological factors, such as electrolyte disorders, drugs, autonomous tone, diseases, and, mainly, heart rate (HR) $[3,4]$. This HR dependence on QT duration was early well established and, consequently, to define normal ranges for QT interval, its actual duration is corrected according to HR [5].

Figure 1 shows other ventricular repolarization intervals that are useful in predicting malignant ventricular arrhythmias. Previous reports on HR dependence on these intervals in normal subjects have indicated that at rest, JT, QTpeak (QTp) and JTpeak (JTp) depend on HR but Tpeak-Tend (Tpe) does not [6-9]. During exercise, QTp and JTp depend on HR and Tpe has a weak but statistically significant dependence on $\operatorname{HR}[6,8,10]$. There are no known reports about the dependence of HR on JT.

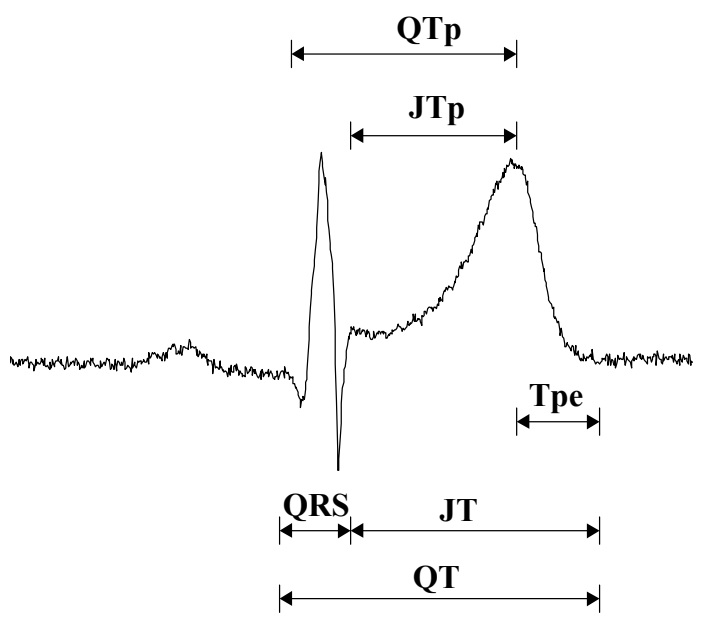

Fig. 1. Time intervals of ventricular repolarization activity.

Dynamics of the ratios between some ventricular repolarization intervals during exercise in normal subjects has also received some attention. It has shown that Tpe/QT increases [11] and QTp/QT decreases [12] at elevated HR, but dynamics of JTp/JT, Tpe/JTp, and Tpe/JT does not seem to have been studied. The aim of this work is to analyze the influence of HR on QTp/QT, $\mathrm{JTp} / \mathrm{JT}$, Tpe/JTp, and Tpe/JT during exercise. These ratios were calculated for the V3 lead recorded on a healthy subject during the first four stages of the Bruce protocol, in two ranges: HR below 100 beats/min and HR from 100 beats $/ \mathrm{min}$ to 185 beats $/ \mathrm{min}$. The characteristic points of QRS and T waves were detected by computer algorithms based on the wavelet transform (WT).

\section{METHODOLOGY}

Subject and data acquisition

The subject was a 45-year old healthy male. Exercise tests were performed on a treadmill (Technogym Run Race HC1200) that can be programmed to follow different medical protocols and includes a wireless heart rate meter which we used to assess that HR did not exceed 185 beats/min. The treadmill was programmed according to the Bruce protocol [13], which includes seven graded steps lasting three minutes each and differing by an increased floor elevation and speed. Signals were acquired by using the modified 12-lead ECG proposed by Mason and Likar [14] for exercise testing. The twelve simultaneous signals were amplified (programmable gain 
$500-2500$, bandwidth from $0.025 \mathrm{~Hz}$ to $500 \mathrm{~Hz}$ ) by a proprietary 16-channel high-resolution system [15] connected to a digitizing plug-in PC card (Data Translation DT21EZ, $12 \mathrm{~b}$ resolution) that sampled them at $1 \mathrm{kHz}$ rate. The analyzed signals were 30 minutes recordings from the V3 lead corresponding to the first four stages of the Bruce protocol.

Data processing and analysis

Alvarado [16] describes the algorithms used to detect characteristics points of the QRS complex and the T wave which are based on WT. The validation process of the three designed algorithms has been performed by using 25 records of the well-annotated reference database for ECG measurement, the CSE multi-lead measurement database [17], in which the golden standard had been derived by an international group of cardiologists who visually determined the on- and -offset points of P, QRS and T waves [18]. In those 15-lead records, the earliest onset (QRS) and the latest offsets (QRS and T wave) of each record were detected, and the measurements were within the tolerance limits for deviations with respect of the manual measurements determined by the CSE experts [19].

When the $\mathrm{T}$ and $\mathrm{P}$ waves merged (approximately at heart rates above 150 beats/min), the T-wave end was estimated as described by Alvarado et al. [16]. The ECG intervals measured from the V3 lead were: QT, QTp, JT, JTp, Tpe, and QRS (Figure 1). Beats displaying a too small T-wave amplitude, or that were severely corrupted by movement artifacts, were excluded. The dependence between the ventricular repolarization interval ratios considered and HR were analyzed by linear regression using the least squares method for two ranges of HR: below 100 beats/min and from 100 to 185 beats/min.

\section{RESULTS AND DISCUSSION}

Figures 2(a) and 2 (b) respectively show QTp/QT and $\mathrm{JTp} / \mathrm{JT}$ during exercise. Table 1 sums up the results of the regression analysis of these ratios versus HR for the two defined HR ranges.

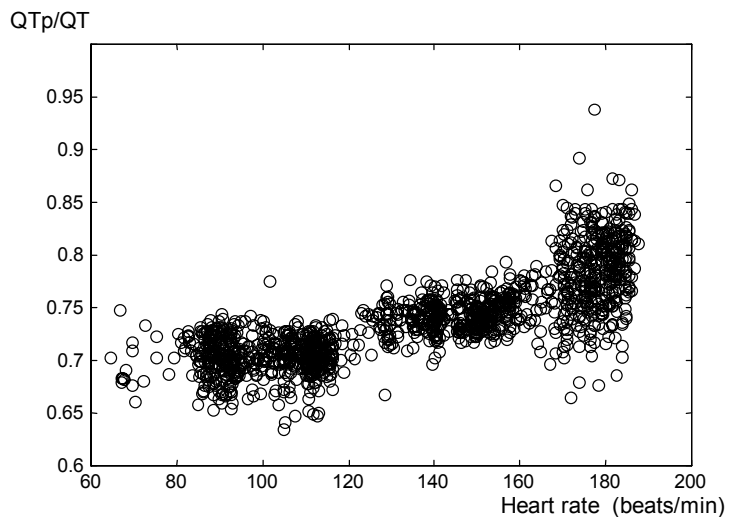

(a)

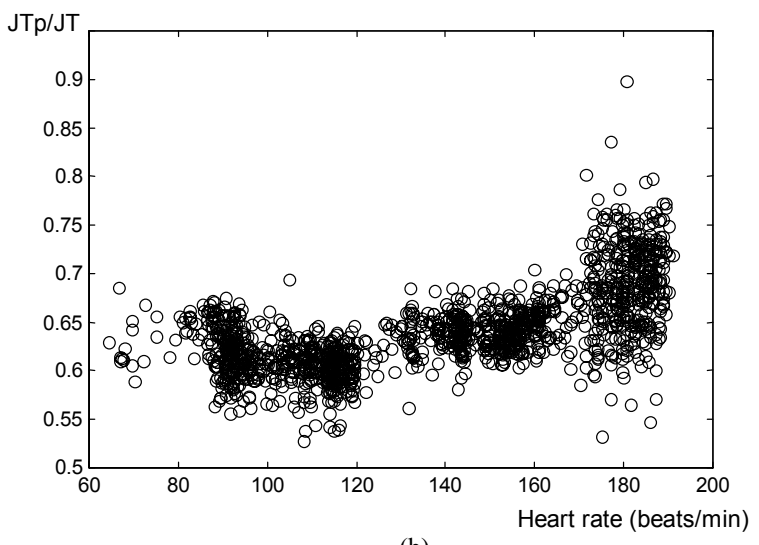

(b)

Fig. 2. Dynamics of (a) QTp/QT and (b) JTp/JT during exercise testing on a healthy subject.

Table 1 Relationship between QTp/QT, JTp/JT and HR ( $m=$ slope, $r=$ correlation coefficient)

\begin{tabular}{|c|c|c|c|c|}
\hline \multirow{2}{*}{} & \multicolumn{2}{|c|}{$\mathrm{HR}<100$ beats/min } & \multicolumn{2}{c|}{ HR $>100$ beats/min } \\
\cline { 2 - 5 } & $m$ & $r$ & $m$ & $R$ \\
\hline $\mathrm{QTp} / \mathrm{QT}$ & 0 & 0.01 & 0.001 & 0.77 \\
\hline $\mathrm{JTp} / \mathrm{JT}$ & 0 & -0.07 & 0.001 & 0.71 \\
\hline
\end{tabular}

When HR $<100$ beats/min, neither QTp/QT nor JTp/JT depend on HR. When HR > 100 beats/min, both QTp/QT and $\mathrm{JTp} / \mathrm{JT}$ increase with HR, and the correlation is high. The increase of QTp/QT with HR disagrees with a previous report [12] describing a decrease of QTp/QT for elevated HR, but in that study [12] method measurement of end of $\mathrm{T}$ wave and HR range measured is unknown, therefore it is not possible compare both studies in elevated HR.

Figures 3(a) and 3(b) respectively show Tpe/JT and Tpe/JTp during exercise. Table 2 sums up the corresponding results of the regression analysis. When HR $<100$ beats/min, neither Tpe/JT nor Tpe/JTp depend on HR. When HR $>100$ beats/min, both Tpe/JT and Tpe/JTp decrease with HR, and the correlation is high.

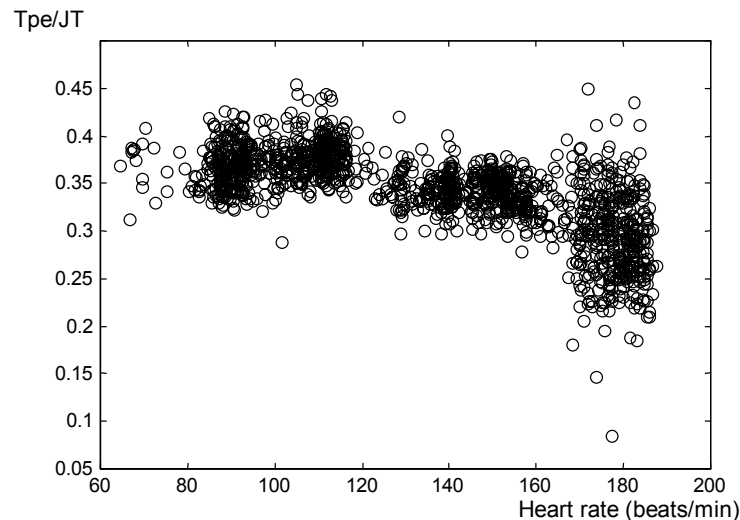

(a) 


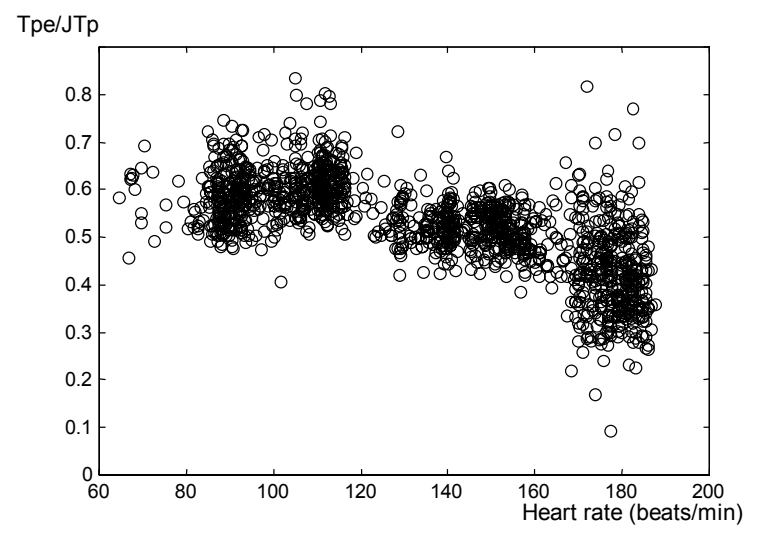

(b)

Fig. 3. Dynamics of (a) Tpe/JT and (b) Tpe/JTp during

exercise testing on a healthy subject.

Table 2. Relationship between Tpe/JT, Tpe/JTp and HR ( $m=$ slope, $r=$ correlation coefficient)

\begin{tabular}{|c|c|c|c|c|}
\hline & \multicolumn{2}{|c|}{$\mathrm{HR}<100$ beats/min } & \multicolumn{2}{c|}{ HR $>100$ beats/min } \\
\cline { 2 - 5 } & $m$ & $r$ & $m$ & $r$ \\
\hline $\mathrm{Tpe} / \mathrm{JT}$ & 0 & 0.07 & -0.001 & -0.71 \\
\hline $\mathrm{Tpe}_{\mathrm{JT}}$ & 0 & 0.07 & -0.002 & -0.72 \\
\hline
\end{tabular}

Therefore, as opposed to the QT interval, there is no need to correct QTp/QT, JTp/JT, Tpe/JTp, and Tpe/JT when $\mathrm{HR}$ is below 100 beats $/ \mathrm{min}$. This independence may increase the importance of those ratios as possible predictors of malignant ventricular arrhythmias and sudden cardiac death. Future work will focus on corroborate these findings in a larger population of postMI patients with and without susceptibility to ventricular tachyarrhythmias.

\section{ACKNOWLEDGEMENT}

This work has been funded by a scholarship from the CONACYT (México) to Carlos Alvarado and by project SAF98-0120 from the Spanish CYCIT. The authors are grateful to J. Amorós for their help in using the treadmill, and to the volunteers, particularly B. Pérez and M. Vargas.

\section{REFERENCES}

[1] B. Surawicz, S.B. Knoebel, "Long QT: good, bad or indifferent?,” J. Am. Coll. Cardiol., vol. 4, pp. 398-413, 1984.

[2] P. Davey, "QT interval and mortality from coronary artery disease," Prog. Cardiovasc. Dis., vol. 42, pp. 359-384, 2000.

[3] C. Funck-Brentano, P. Jaillon, "Rate-corrected QT interval: techniques and limitations," Am. J. Cardiol., vol. 72 , no. 6, pp 17B-22B, 1993.
[4] A.J. Moss, "Measurement of the QT interval and the risk associated with QTc interval prolongation: a review," Am. J. Cardiol., vol. 72, no. 6, pp. 23B-25B, 1993.

[5] H.C. Bazett, "An analysis of the time-relations of electrocardiograms," Heart, vol. 7, pp. 353-370, 1920.

[6] J. O’Donnell, S.B. Knoebel, D.E. Lovelace, P.L. McHenry, "Computer quantitation of Q-T and terminal $\mathrm{T}$ wave (aT-eT) intervals during exercise: methodology and results in normal men," Am. J. Cardiol., vol. 47, pp. 1168-1172, 1981.

[7] M. Merri, J. Benhorin, M. Alberti, E. Locati, A.J. Moss, "Electrocardiographic quantitation of ventricular repolarization," Circulation, vol. 80, pp. 1301-1308, 1989.

[8] K.G. Lax, P.M. Okin, P. Kligfield, "Electrocardiographic repolarization measurements at rest and during exercise in normal subjects and in patients with coronary artery disease," Am. Heart J. vol. 128, pp. 271-280, 1994.

[9] I. Savelieva, S.B. Reddy, A.J. Camm, M. Malik, "Does dispersion of repolarization depend on cardiac cycle length and should it be rate-corrected? Observations in 1906 healthy subjects (abstract)," J. Am. Coll. Cardiol., vol. 35, no. 2, Suppl. A, pp. 143, 2000.

[10] P.P. Davey, "QT interval measurement: Q to Tapex or Q to Tend," J. Int. Med., 246, pp. 145-149, 1999.

[11] J. O'Donnell, D.E. Lovelace, S.B. Knoebel, P.L. McHenry, "Behavior of the terminal $\mathrm{T}$ wave during exercise in normal subjects, patients with symptomatic coronary artery disease and apparently healthy subjects with abnormal ST segment depression," J. Am. Coll. Cardiol., vol. 5, pp. 78-84, 1985.

[12] L. Zhang, G.M. Vincent, "Sympathetic modulation affects repolarization disparity in LQTS and normals: QTp/QTe changes during exercise and B-blockade (abstract), J. Am. Coll. Cardiol. (Feb. Special Issue), pp. 37A, 1994.

[13] R.A. Bruce, F. Kusumi, D. Hosmer, "Maximal oxygen intake and nomographic assessment of functional aerobic impairment in cardiovascular disease," Am. Heart J., vol. 85, pp. 546-562, 1973.

[14] R.E. Mason, I. Likar, "A new system of multiple-lead exercise electrocardiography," Am. Heart J., vol. 71, no. 2, pp. 196-205, 1966.

[15] J. Ramos, "Detección de micropotenciales auriculares de alta frecuencia," Ph.D. dissertation, Technical University of Catalonia, Barcelona, Spain, 1997.

[16] C. Alvarado, "Análisis de la variabilidad de los intervalos de tiempo del ECG," Ph.D. dissertation, Technical University of Catalonia, Barcelona, Spain, 2001.

[17] Willems J.L., P. Arnaud, J.H. Van Bemmel, R. Degani, P.W. Macfarlane, C. Zywietz for the CSE Working Party, "Common standards for quantitative electrocardiography: goals and main results," Meth. Inform. Med., vol 29, no. 263, 1990

[18] J.L. Willems: Common standards for quantitative electrocardiography. CSE multilead atlas, Measurement resultsdata set 3. CSE Project. ACCO Publ., Leuven, Belgium, 1988.

[19] The CSE working party, "Recommendations for measurement standards in quantitative electrocardiography," Eur. Heart J., vol. 6, pp. 815-825, 1985. 